\title{
Synthesis of the Key Intermediate, Diethyl 2-Acetylamino-2-(2-(4- octanoylphenyl)ethyl)propane-1,3-dioate, of the Immunomodulatory Agent FTY720 (Fingolimod)
}

\author{
Norimasa Matsumoto, ${ }^{a, 1)}$ Ryoji Hirose, ${ }^{a, 2)}$ Shigeo Sasaki, ${ }^{a, 3)}$ and Tetsuro FujitA ${ }^{*, b, 4)}$ \\ ${ }^{a}$ Research Laboratory, Taito Co., Ltd.; 1-26 Higashi Shiriike-Shinmachi, Nagata-ku, Kobe 653-0023, Japan: and ${ }^{b}$ Faculty \\ of Pharmaceutical Sciences, Setsunan University; 45-1 Nagaotoge-cho, Hirakata, Osaka 573-0101, Japan. \\ Received October 26, 2007; accepted January 18, 2008; published online January 22, 2008
}

\begin{abstract}
The key intermediate, diethyl 2-acetylamino-2-(2-(4-octanoylphenyl)ethyl)propane-1,3-dioate (13), for the immunomodulatory agent FTY720 (2: fingolimod) was synthesized via Michael addition of diethyl(acetylamino)malonate (6) to 4-octanoylstyrene (12).
\end{abstract}

Key words FTY720; immunomodulatory agent; Michael addition; fingolimod

Members of our group previously isolated a potent immunosuppressant, ISP-I (1) (Chart 1), from the culture broth of Isaria sinclairii (ATCC24400), and showed that it could suppress both lymphocyte proliferation in the mouse allogeneic mixed lymphocyte reaction (MLR) system in vitro and generation of allo-reactive cytotoxic $\mathrm{T}$ lymphocytes in mice in vivo. ${ }^{5}$ Compound $\mathbf{1}$ is identical with the antifungal antibiotics myriocin $^{6,7)}$ and thermozymocidin ${ }^{8,9)}$ which are produced by Myriococcum albomyces and Mycelia sterilia, respectively. The structure of $\mathbf{1}$ was subsequently modified in order to reduce its toxicity, to improve its physicochemical properties and to identify the essential structure for immunosuppressive activity ${ }^{10)}$ and a candidate compound, FTY720 (2: 2-amino-2-(2-(4-octylphenyl)ethyl)propane-1,3-diol hydrochloride, fingolimod), was obtained. ${ }^{11-14)}$ Compound 2 does not impair the activation, proliferation or effector functions of T- and B-cells, in contrast with established immunosuppressants (cyclosporin, tacrolimus, etc.). It has been proposed that $\mathbf{2}$ is phosphorylated by sphingosine kinase, and the product, FTY720 monophosphate, acts as an agonist of sphingosine-1-phosphate (S1P) and modulates immune response by sequestering lymphocytes from peripheral blood to the secondary lymphoid tissues. ${ }^{15-18)}$ Thus, 2 has a unique mechanism of action and is expected to be useful for immunosuppression following organ transplantation and for the treatment of various diseases, including multiple sclerosis, ${ }^{16)}$ rheumatoid arthritis, ${ }^{19)}$ atopic dermatitis, ${ }^{20)}$ and myasthenia gravis, ${ }^{21)}$ based on findings in animal models. Clinical trials of 2 in renal transplantation ${ }^{22-26)}$ and multiple sclerosis patients $^{27)}$ have been performed.

Adachi et al. first synthesized 2 via the route $\mathbf{3} \rightarrow \mathbf{4} \rightarrow \rightarrow$ $\rightarrow \rightarrow \mathbf{5} \rightarrow \mathbf{7} \rightarrow \mathbf{2}$, as shown Chart 2, in cooperation with our

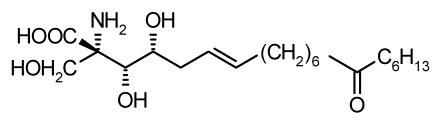

ISP-I (1: myriocin, thermozymocidin)

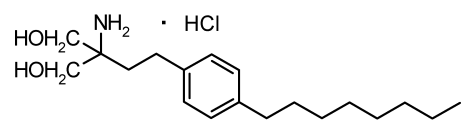

FTY720 (2: fingolimod)

Chart 1 group. ${ }^{11,12,28)}$ We subsequently patented a more convenient synthesis of 2 via routes including Michael addition $(3 \rightarrow \mathbf{4} \rightarrow$ $8 \rightarrow \mathbf{9} \rightarrow[\mathbf{1 2}] \rightarrow \mathbf{1 3} \rightarrow \mathbf{7} \rightarrow \mathbf{2}, \quad \mathbf{1 0} \rightarrow \mathbf{1 1} \rightarrow \mathbf{1 3} \rightarrow \mathbf{7} \rightarrow \mathbf{2}){ }^{29)}$ Other synthetic routes to 2 were subsequently developed. ${ }^{30-33)}$ Here, we describe the convenient synthesis of the key intermediate, diethyl 2-acetylamino-2-(2-(4-octanoylphenyl)ethyl)propane1,3-dioate (13) in detail.

To improve Adachi's method, we focused on the condensation of 5 and $\mathbf{6}$. During this condensation, nucleophilic substitution occurs as the main reaction and elimination as a side reaction to afford 7 and the styrene derivative, respectively. Therefore, 5 was replaced with $4^{\prime}$-(2-haloethyl)octanophenone. Even if 4'-(2-haloethyl)octanophenone affords the styrene $\mathbf{1 2}^{34)}$ by elimination, it is expected that $\mathbf{1 2}$ will undergo Michael-type nucleophilic attack by the anion of the malonate $\mathbf{6}$ to give the intermediate 13. Further, the synthesis can be shortened, because 4'-(2-haloethyl)octanophenone can be derived from phenethyl halide, such as phenethyl bromide (10). First, the iodide 9 was synthesized from the acetate 4 by deprotection and iodination, and condensed with 6 to afford 13. At the same time, it was found that hydrogen iodide was eliminated from 9 to give the styrene $\mathbf{1 2}$ (equivalent to half the total amount of product), which was condensed with 6 to give 13 by Michael addition reaction. Next, the bromide $\mathbf{1 1}^{34)}$ derived from $\mathbf{1 0}$ was examined. It was found that the elimination and condensation reactions could be run in one pot (see Experimental) to afford the key intermediate 13, which was hydrogenated to Adachi's intermediate, 7.

In conclusion, we have developed a convenient synthesis of the key intermediate, diethyl 2-acetylamino-2-(2-(4-octanoylphenyl)ethyl)propane-1,3-dioate (13), of FTY720 (2: fingolimod) from phenethyl bromide (10) in $55 \%$ overall yield. Adachi's method provided the common intermediate, 7, from 3 in 6 steps with $18 \%$ yield, ${ }^{11,12)}$ while the new method afforded 7 from 10 (via 13) in only 3 steps with $41 \%$ yield.

\section{Experimental}

General Information Melting points were determined on a Yanagimoto micro melting point apparatus without correction. IR spectra were taken on a Shimadzu FTIR-8400 infrared spectrophotometer. Mass spectra (EI-MS, FAB-MS) were taken on a Shimadzu QP-2000 and JEOL JMS-700T spectrometers. ${ }^{1} \mathrm{H}-\mathrm{NMR}$ spectra were taken on a JEOL EX-270, JEOL JNMGX400, and Varian UNITY plus 500 spectrometers with TMS as an internal standard; chemical shifts are reported in parts per million. TLC was performed on aluminum-seated silica gel $60 \mathrm{~F}_{254}$ (Merck). For column chro- 


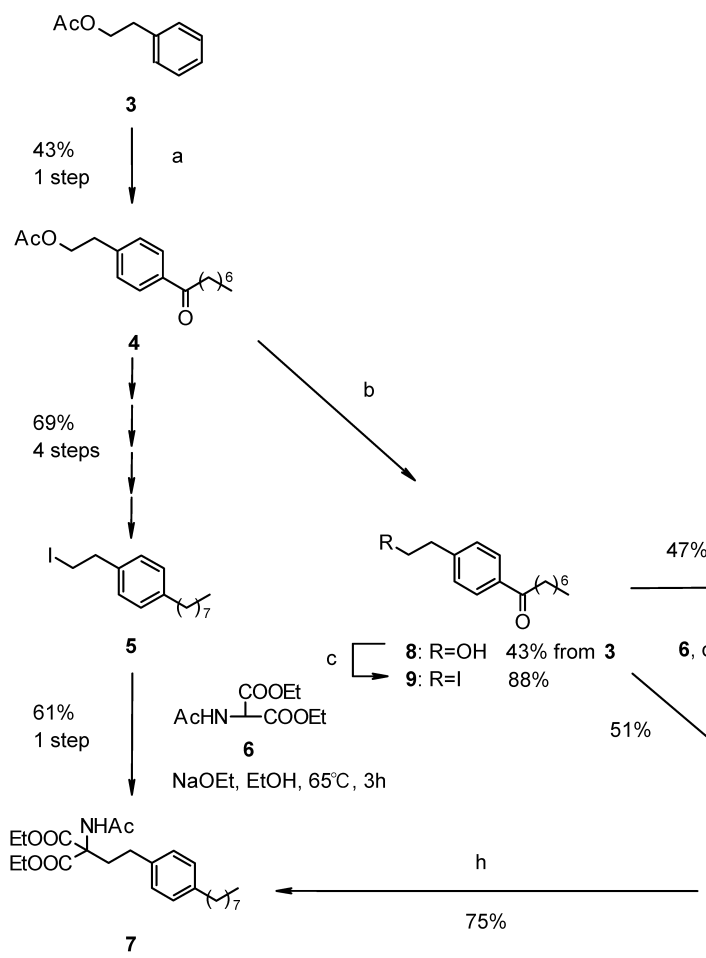

$18 \%$ from 3 via 5 (Adachi's method) $25 \%$ from 3 via 9 $41 \%$ form 10

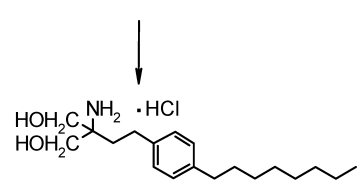<smiles>BrCCc1ccccc1</smiles>

10<smiles>C1CCC1</smiles>

,<smiles>CC(C)C(=O)c1ccc(CCBr)cc1</smiles>

11

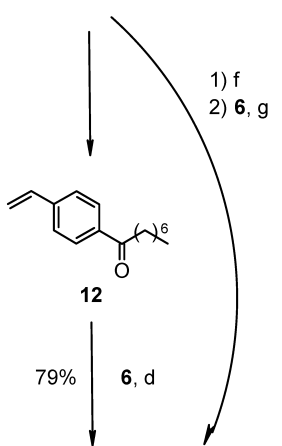<smiles>CCOC(=O)c1ccc(CC(C)(NC(C)=O)OCC)cc1</smiles>

$33 \%$ from 3 $55 \%$ from 10

FTY720 (2)

Reagents and conditions: (a) $\mathrm{C}_{7} \mathrm{H}_{15} \mathrm{COCl}, \mathrm{AlCl}_{3}, 1$,2-dichloroethane, rt, 2.5h; (b) $28 \% \mathrm{NaOMe}$ in $\mathrm{MeOH}, \mathrm{MeOH}$, rt, $1 \mathrm{~h}$; (c) $(\mathrm{Ph})_{3} \mathrm{P}$, imidazole, EtOAc, rt, $1 \mathrm{~h}$; (d) $60 \% \mathrm{NaH}$, DMF, $60^{\circ} \mathrm{C}, 2-6 \mathrm{~h}$; (e) $\mathrm{C}_{7} \mathrm{H}_{15} \mathrm{COCl}, \mathrm{AlCl}_{3}, \mathrm{CH}_{2} \mathrm{Cl}_{2}$, rt, overnight; (f) $\mathrm{NaOEt}$, EtOH, $60^{\circ} \mathrm{C}, 1 \mathrm{~h}$; (g) DMF, $60^{\circ} \mathrm{C}$, overnight; (h) $\mathrm{Pd} / \mathrm{C}, \mathrm{H}_{2}$, EtOH, overnight

\section{Chart 2}

matography, silica gel ( $70-230$ mesh, Merck) was used. Organic solvent extracts were dried over anhydrous magnesium sulfate, and evaporation of solvents was performed under reduced pressure.

4'-(2-Hydroxyethyl)octanophenone (8) To a solution of octanoyl chloride $(216 \mathrm{~g}, 1.33 \mathrm{~mol})$ and phenethyl acetate $(3,285 \mathrm{~g}, 1.74 \mathrm{~mol})$ in $1,2-$ dichloroethane $(900 \mathrm{ml})$ was added $\mathrm{AlCl}_{3}(372 \mathrm{~g}, 2.80 \mathrm{~mol})$ under ice cooling. The mixture was stirred at room temperature for $2.5 \mathrm{~h}$, then poured into water. The extracted organic solution was washed with water, dried, and evaporated. The residue was dried in vacuo to give a colorless oil $(280 \mathrm{~g})$ containing 2-(4-octanoylphenyl)ethyl acetate $\mathbf{4}$ as the major component. To this oil $(280 \mathrm{~g})$ in $\mathrm{MeOH}(200 \mathrm{ml})$ was added $28 \%$ sodium methoxide $(5.3 \mathrm{~g}$, $82.8 \mathrm{mmol})$ in $\mathrm{MeOH}(18.8 \mathrm{ml})$, and the mixture was stirred at room temperature for $1 \mathrm{~h}$. A suspension of Amberlite IR-120B in $\mathrm{MeOH}(98 \mathrm{ml})$ was added to the reaction mixture, and removed by filtration, then the filtrate was evaporated. The residue was recrystallized from $n$-hexane-EtOAc $(10: 1)$ to give $8(138 \mathrm{~g}, 43 \%)$ as colorless plates: $\mathrm{mp} 47.4^{\circ} \mathrm{C}$; IR $v_{\max }(\mathrm{KBr}) \mathrm{cm}^{-1}$ : 3260, 2910, 2850, 1680; ${ }^{1} \mathrm{H}-\mathrm{NMR}\left(500 \mathrm{MHz}, \mathrm{CDCl}_{3}\right) \delta: 7.91(2 \mathrm{H}, \mathrm{d}$, $J=8.5 \mathrm{~Hz}, \operatorname{Ar} \underline{\mathrm{H}}), 7.32(2 \mathrm{H}, \mathrm{d}, J=8.5 \mathrm{~Hz}, \operatorname{Ar} \underline{\mathrm{H}}), 3.90(2 \mathrm{H}, \mathrm{t}, J=6.6 \mathrm{~Hz}$, $\left.\mathrm{CH}_{2} \mathrm{OH}\right), 2.94\left(2 \mathrm{H}, \mathrm{t}, J=7.3 \mathrm{~Hz}, \mathrm{COCH}_{2}\right), 2.93\left(2 \mathrm{H}, \mathrm{t}, J=6.6 \mathrm{~Hz}, \mathrm{PhCH}_{2}\right)$, $1.72\left(2 \mathrm{H}\right.$, quintet, $\left.J=7.3 \mathrm{~Hz}, \mathrm{COCH}_{2} \underline{\mathrm{CH}}_{2}\right), 1.59(1 \mathrm{H}$, br s, OH), $1.40-1.26$ $\left(8 \mathrm{H}, \mathrm{m},\left(\mathrm{CH}_{2}\right)_{4} \mathrm{CH}_{3}\right), 0.88\left(3 \mathrm{H}, \mathrm{t}, J=7.1 \mathrm{~Hz}, \mathrm{CH}_{3}\right)$; EI-MS $m / z: 248\left([\mathrm{M}]^{+}\right)$.

4'-(2-Iodoethyl)octanophenone (9) To a solution of $8(137 \mathrm{~g}, 0.55$ $\mathrm{mol})$, imidazole $(53 \mathrm{~g}, 0.78 \mathrm{~mol})$, and triphenylphosphine $(174 \mathrm{~g}, 0.66 \mathrm{~mol})$ in EtOAc $(550 \mathrm{ml})$ was added iodine $(197 \mathrm{~g}, 0.78 \mathrm{~mol})$ under ice cooling. The mixture was stirred at room temperature for $1 \mathrm{~h}$, then diluted with EtOAc, washed with saturated $\mathrm{NaHCO}_{3}$ aqueous solution and brine, dried and evaporated. The residue was suspended in $n$-hexane-EtOAc $(10: 1)$, and filtered through silica gel, and the eluate was evaporated to give 9 (175 g, $88 \%$ ) as a white solid: $\mathrm{mp} 36.5^{\circ} \mathrm{C}$; IR $v_{\max }(\mathrm{KBr}) \mathrm{cm}^{-1}: 2950,2920,2850$, $1680,1600,1230 ;{ }^{1} \mathrm{H}-\mathrm{NMR}\left(500 \mathrm{MHz}, \mathrm{CDCl}_{3}\right) \delta: 7.90(2 \mathrm{H}, \mathrm{d}, J=8.3 \mathrm{~Hz}$, $\operatorname{Ar} \underline{\mathrm{H}}), 7.26(2 \mathrm{H}, \mathrm{d}, J=8.3 \mathrm{~Hz}, \mathrm{Ar} \underline{\mathrm{H}}), 3.35\left(2 \mathrm{H}, \mathrm{t}, J=7.3 \mathrm{~Hz}, \mathrm{CH}_{2} \mathrm{I}\right), 3.22(2 \mathrm{H}$, $\left.\mathrm{t}, J=7.3 \mathrm{~Hz}, \mathrm{PhCH}_{2}\right), 2.92\left(2 \mathrm{H}, \mathrm{t}, J=7.6 \mathrm{~Hz}, \mathrm{COCH}_{2}\right), 1.71$ (2H, quintet, $\left.J=7.1 \mathrm{~Hz}, \mathrm{COCH}_{2} \underline{\mathrm{C}}_{2}\right), 1.36-1.25\left(8 \mathrm{H}, \mathrm{m},\left(\mathrm{CH}_{2}\right)_{4} \mathrm{CH}_{3}\right), 0.86(3 \mathrm{H}, \mathrm{t}$, $\left.J=7.1 \mathrm{~Hz}, \mathrm{CH}_{3}\right)$; EI-MS m/z: $274\left(\left[\mathrm{I}\left(\mathrm{CH}_{2}\right)_{2} \mathrm{C}_{6} \mathrm{H}_{4} \mathrm{C}(\mathrm{OH})=\mathrm{CH}_{2}\right]^{+}\right)$. HR-MS (EI) $m / z$ : 358.0789 (Calcd for $\mathrm{C}_{16} \mathrm{H}_{23} \mathrm{OI}: 358.0794$ ). HR-MS (FAB ${ }^{+}$) $m / z$ : 359.0879 (Calcd for $\mathrm{C}_{16} \mathrm{H}_{24} \mathrm{OI}$ : 359.0872).

4-Octanoylstyrene (12) and Diethyl 2-Acetylamino-2-(2-(4-octanoylphenyl)ethyl)propane-1,3-dioate (13) To a solution of diethyl (acetylamino)malonate $(6,9.09 \mathrm{~g}, 41.9 \mathrm{mmol})$ in dried DMF $(30 \mathrm{ml})$ was added $60 \%$ sodium hydride $(1.23 \mathrm{~g}, 30.8 \mathrm{mmol})$ under ice cooling, and the mixture was stirred at room temperature for $1 \mathrm{~h}$ under a nitrogen atmosphere. To this mixture was added $9(5.0 \mathrm{~g}, 14.0 \mathrm{mmol})$ in dried DMF $(15 \mathrm{ml})$ under a nitrogen atmosphere, and the whole was stirred at $60^{\circ} \mathrm{C}$ for $2 \mathrm{~h}$, poured into ice water, and extracted with $\mathrm{Et}_{2} \mathrm{O}$. The extract was washed with brine, dried and evaporated. The residue was chromatographed on silica gel with $n$ hexane to give $12(1.5 \mathrm{~g}, 47 \%)$ as a white powder and with $n$-hexane-EtOAc $(3: 1)$ to give $\mathbf{1 3}(3.2 \mathrm{~g}, 51 \%)$ as a white powder.

13 from 12 To a solution of diethyl(acetylamino)malonate $(6,4.25 \mathrm{~g}$, $19.6 \mathrm{mmol})$ in dried DMF $(30 \mathrm{ml})$ was added $60 \%$ sodium hydride $(0.57 \mathrm{~g}$, $14.3 \mathrm{mmol}$ ) under ice cooling, and the mixture was stirred at room temperature for $0.5 \mathrm{~h}$ under a nitrogen atmosphere. Then $12(1.5 \mathrm{~g}, 6.5 \mathrm{mmol})$ in dried DMF $(15 \mathrm{ml})$ was added under a nitrogen atmosphere. The mixture was stirred at $60^{\circ} \mathrm{C}$ for $6 \mathrm{~h}$, and at room temperature for $2 \mathrm{~d}$, then poured into ice water, and extracted with $\mathrm{Et}_{2} \mathrm{O}$. The extract was washed with brine, 
dried and evaporated. The residue was chromatographed on silica gel with $n$ hexane-EtOAc ( $3: 1)$ to give $\mathbf{1 3}(2.3 \mathrm{~g}, 79 \%)$ as a white powder.

13 from 10 To a solution of phenethyl bromide $(\mathbf{1 0}, 5.00 \mathrm{~g}, 27.0 \mathrm{mmol})$ and octanoyl chloride $(4.83 \mathrm{~g}, 29.1 \mathrm{mmol})$ in $\mathrm{CH}_{2} \mathrm{Cl}_{2}(40 \mathrm{ml})$ was added $\mathrm{AlCl}_{3}(3.67 \mathrm{~g}, 27.6 \mathrm{~mol})$ at $-20^{\circ} \mathrm{C}$. The mixture was stirred at $-20^{\circ} \mathrm{C}$ for $1 \mathrm{~h}$ and at room temperature overnight, then poured into ice water, and extracted with $\mathrm{Et}_{2} \mathrm{O}$. The extract was washed successively with $1 \mathrm{~N} \mathrm{HCl}$, brine saturated $\mathrm{NaHCO}_{3}$ aqueous solution and brine. The extract was dried and evaporated. The residue was dissolved in $n$-hexane-EtOAc $(20: 1)$, and the solution was filtered through silica gel and evaporated to give a pale yellow oil $(6.96 \mathrm{~g})$ containing $4^{\prime}$-(2-bromoethyl)octanophenone (11) as the major component

To this oil $(0.50 \mathrm{~g})$ in dried EtOH $(2 \mathrm{ml})$ was added sodium ethoxide $(0.16 \mathrm{~g}, 2.35 \mathrm{mmol})$ under a nitrogen atmosphere, and the mixture was stirred at $60^{\circ} \mathrm{C}$ for $1 \mathrm{~h}$, and then DMF $(10 \mathrm{ml}), 6(1.05 \mathrm{~g}, 4.84 \mathrm{mmol})$ and sodium ethoxide $(0.25 \mathrm{~g}, 3.68 \mathrm{mmol})$ were added to the mixture under a nitrogen atmosphere. The mixture was stirred at $60{ }^{\circ} \mathrm{C}$ overnight, then poured into ice water, and extracted with $\mathrm{Et}_{2} \mathrm{O}$. The extract was washed with brine, dried, and evaporated. The residue was chromatographed on silica gel with $n$-hexane-EtOAc ( $3: 1)$ to give $\mathbf{1 3}(0.48 \mathrm{~g}, 55 \%)$ as a white powder.

Compound 11: IR $v_{\max }(\mathrm{KBr}) \mathrm{cm}^{-1}: 2960,2930,2860,1690(\mathrm{C}=\mathrm{O})$, $1610,1410,1260,1220,1180 ;{ }^{1} \mathrm{H}-\mathrm{NMR}\left(500 \mathrm{MHz}, \mathrm{CDCl}_{3}\right) \delta: 7.92(2 \mathrm{H}, \mathrm{d}$, $J=8.3 \mathrm{~Hz}, \operatorname{ArH}), 7.30(2 \mathrm{H}, \mathrm{d}, J=8.3 \mathrm{~Hz}, \operatorname{ArH}), 3.59(2 \mathrm{H}, \mathrm{t}, J=7.4 \mathrm{~Hz}$ $\left.\mathrm{BrCH}_{2}\right), 3.22\left(2 \mathrm{H}, \mathrm{t}, J=7.4 \mathrm{~Hz}, \mathrm{BrCH}_{2} \mathrm{CH}_{2}\right), 2.90(2 \mathrm{H}, \mathrm{t}, J=7.4 \mathrm{~Hz}$, $\left.\mathrm{COCH}_{2}\right), 1.73\left(2 \mathrm{H}\right.$, quintet, $\left.J=7.4 \mathrm{~Hz}, \mathrm{COCH}_{2} \mathrm{CH}_{2}\right), 1.38-1.27(8 \mathrm{H}, \mathrm{m}$, $\left.\left(\mathrm{CH}_{2}\right)_{4} \mathrm{CH}_{3}\right), 0.88\left(3 \mathrm{H}, \mathrm{t}, J=7.1 \mathrm{~Hz}, \mathrm{CH}_{3}\right)$; EI-MS $m / z: 312$ and $310\left([\mathrm{M}]^{+}\right)$.

Compound 12: mp $60.5^{\circ} \mathrm{C}$ (lit. ${ }^{34)} \mathrm{mp} 62-63^{\circ} \mathrm{C}$ ). IR $v_{\max }(\mathrm{KBr}) \mathrm{cm}^{-1}$ : 2920, 2850, 1670, 1470, 1410, 1320, 1280, 990, 910, 860; ${ }^{1} \mathrm{H}-\mathrm{NMR}$ $\left(400 \mathrm{MHz}, \mathrm{CDCl}_{3}\right) \delta: 7.92(2 \mathrm{H}, \mathrm{d}, J=8.3 \mathrm{~Hz}, \mathrm{ArH}), 7.47(2 \mathrm{H}, \mathrm{d}, J=8.3 \mathrm{~Hz}$ $\mathrm{Ar} \underline{\mathrm{H}}), 6.75\left(1 \mathrm{H}, \mathrm{dd}, J=17.6,10.9 \mathrm{~Hz}, \mathrm{CHCH}_{2}\right), 5.86(1 \mathrm{H}, \mathrm{d}, J=17.7 \mathrm{~Hz}$, $\left.\mathrm{CH}_{2}=\right), 5.38\left(1 \mathrm{H}, \mathrm{d}, J=10.9 \mathrm{~Hz}, \mathrm{CH}_{\mathrm{b}}=\right), 2.90\left(2 \mathrm{H}, \mathrm{t}, J=7.3 \mathrm{~Hz}, \mathrm{COCH}_{2}\right)$ $1.73\left(2 \mathrm{H}\right.$, quintet, $\left.J=7.3 \mathrm{~Hz}, \mathrm{COCH}_{2} \mathrm{CH}_{2}\right), 1.35-1.29\left(8 \mathrm{H}, \mathrm{m},\left(\mathrm{CH}_{2}\right)_{4} \mathrm{CH}_{3}\right)$, $0.88\left(3 \mathrm{H}, \mathrm{t}, J=6.8 \mathrm{~Hz}, \mathrm{CH}_{3}\right)$; EI-MS $m / z: 230\left([\mathrm{M}]^{+}\right)$.

Compound 13: $\mathrm{mp} 79.0^{\circ} \mathrm{C}$; IR $v_{\max }(\mathrm{KBr}) \mathrm{cm}^{-1}$ : 3250, 2930, 2850, 1750, $1680,1650,1520,1260,1220,1200 ;{ }^{1} \mathrm{H}-\mathrm{NMR}\left(500 \mathrm{MHz}, \mathrm{CDCl}_{3}\right) \delta: 7.84$ $(2 \mathrm{H}, \mathrm{d}, J=8.3 \mathrm{~Hz}, \mathrm{Ar} \underline{\mathrm{H}}), 7.21(2 \mathrm{H}, \mathrm{d}, J=8.3 \mathrm{~Hz}, \mathrm{Ar} \underline{\mathrm{H}}), 6.75(1 \mathrm{H}, \mathrm{br} \mathrm{s}, \mathrm{N} \underline{\mathrm{H}})$, $4.20\left(2 \mathrm{H}, \mathrm{q}, J=6.8 \mathrm{~Hz}, \mathrm{OCH}_{2} \mathrm{CH}_{3}\right), 4.19\left(2 \mathrm{H}, \mathrm{q}, J=7.1 \mathrm{~Hz}, \mathrm{OCH}_{2} \mathrm{CH}_{3}\right), 2.90$ $\left(2 \mathrm{H}, \mathrm{t}, J=7.3 \mathrm{~Hz}, \mathrm{COC}_{2}\right), 2.69\left(2 \mathrm{H}, \mathrm{m}, \mathrm{PhCH}_{2}\right), 2.51\left(2 \mathrm{H}, \mathrm{m}, \mathrm{PhCH}_{2} \mathrm{C}_{2}\right)$, $1.96\left(3 \mathrm{H}, \mathrm{s}, \mathrm{NHCOCH}_{3}\right), 1.69\left(2 \mathrm{H}\right.$, quintet, $\left.J=7.3 \mathrm{~Hz}, \mathrm{COCH}_{2} \mathrm{CH}_{2}\right), 1.32$ $\left(2 \mathrm{H}, \mathrm{m}, \mathrm{CO}\left(\mathrm{CH}_{2}\right)_{2} \mathrm{CH}_{2}\right), 1.27\left(6 \mathrm{H}, \mathrm{m},\left(\mathrm{CH}_{2}\right)_{3} \mathrm{CH}_{3}\right), 1.23(6 \mathrm{H}, \mathrm{t}, J=$ $\left.7.1 \mathrm{~Hz}, \quad \mathrm{OCH}_{2} \mathrm{CH}_{3} \times 2\right), 0.86\left(3 \mathrm{H}, \mathrm{t}, J=6.8 \mathrm{~Hz}, \mathrm{CH}_{3}\right) ;$ EI-MS $\mathrm{m} / \mathrm{z}: 402$ $\left(\left[\mathrm{M}-\mathrm{OCH}_{2} \mathrm{CH}_{3}\right]^{+}\right.$). HR-MS (EI) $\mathrm{m} / z$ : 447.2614 (Calcd for $\mathrm{C}_{25} \mathrm{H}_{37} \mathrm{NO}_{6}$ : 447.2620).

Diethyl 2-Acetylamino-2-(2-(4-octylphenyl)ethyl)propane-1,3-dioate (7) A solution of $\mathbf{1 3}(923 \mathrm{~g}, 2.06 \mathrm{~mol})$ in EtOH (101) was stirred under a hydrogen atmosphere in the presence of $5 \% \mathrm{Pd} / \mathrm{C}(138 \mathrm{~g})$ overnight. The catalyst was removed by filtration and the filtrate was evaporated to give a residue. This was recrystallized from $n$-hexane to give $7(670 \mathrm{~g}, 75 \%)$, which was identical with an authentic sample of $7^{12)}$ (IR, ${ }^{1} \mathrm{H}-\mathrm{NMR}, \mathrm{MS}$ ), as a white powder: $\mathrm{mp} 61.0^{\circ} \mathrm{C}$; IR $v_{\max }(\mathrm{KBr}) \mathrm{cm}^{-1}: 3300,2920,2850,1750,1650$, $1520,1220,1200 ;{ }^{1} \mathrm{H}-\mathrm{NMR}\left(270 \mathrm{MHz}, \mathrm{DMSO}-d_{6}\right) \delta: 8.32(1 \mathrm{H}, \mathrm{br} \mathrm{s}, \mathrm{NH})$, $7.08(2 \mathrm{H}, \mathrm{d}, J=7.9 \mathrm{~Hz}, \operatorname{Ar} \underline{\mathrm{H}}), 7.02(2 \mathrm{H}, \mathrm{d}, J=7.9 \mathrm{~Hz}, \operatorname{Ar} \underline{\mathrm{H}}), 4.13(4 \mathrm{H}, \mathrm{q}$, $\left.J=7.3 \mathrm{~Hz}, \mathrm{OCH}_{2} \mathrm{CH}_{3} \times 2\right), 2.52\left(4 \mathrm{H}, \mathrm{m}, J=7.3 \mathrm{~Hz}, \mathrm{PhCH}_{2} \times 2\right), 2.37(2 \mathrm{H}, \mathrm{m}$, $\left.\mathrm{PhCH}_{2} \mathrm{CH}_{2} \mathrm{C}\right), 1.94\left(3 \mathrm{H}, \mathrm{s}, \mathrm{NHCOCH}_{3}\right), 1.52\left(2 \mathrm{H}, \mathrm{m}, \mathrm{PhCH}_{2} \mathrm{CH}_{2}\right), 1.24$ $\left(10 \mathrm{H}, \mathrm{m},\left(\mathrm{CH}_{2}\right)_{5} \mathrm{CH}_{3}\right), 1.15\left(6 \mathrm{H}, \mathrm{t}, J=7.3 \mathrm{~Hz}, \mathrm{OCH}_{2} \mathrm{CH}_{3} \times 2\right), 0.85(3 \mathrm{H}, \mathrm{t}$, $\left.J=6.6 \mathrm{~Hz}, \mathrm{CH}_{3}\right)$; EI-MS $m / z: 433\left([\mathrm{M}]^{+}\right), 388\left(\left[\mathrm{M}-\mathrm{OCH}_{2} \mathrm{CH}_{3}\right]^{+}\right)$; Anal. Calcd for $\mathrm{C}_{25} \mathrm{H}_{39} \mathrm{NO}_{5}: \mathrm{C}, 69.25 ; \mathrm{H}, 9.06 ; \mathrm{N}, 3.23 ; \mathrm{O}, 18.45$. Found: $\mathrm{C}, 69.48$; $\mathrm{H}, 8.76 ; \mathrm{N}, 3.21 ; \mathrm{O}, 18.55$.

Acknowledgment The authors are grateful to Dr. S. Yamaguchi of the Faculty of Pharmaceutical Sciences, Setsunan University for measurement of the mass spectra of compounds $\mathbf{9}$ and $\mathbf{1 3}$.

\section{References and Notes}

1) Present address: Fukuoka Factory, Production Group, Mitsui Sugar Co., Ltd.; 6-11-30 Hakozakifuto, Higashi-ku, Fukuoka 812-0051, Japan.

2) Present address: Quality Assurance Division, Production Group, Mitsui Sugar Co., Ltd.; 2-8-2 Nihonbashi-Honcho, Chuo-ku, Tokyo 103-8423, Japan

3) Present address: Research Laboratory, Mitsui Sugar Co., Ltd.; 1-2-14 Honson, Chigasaki, Kanagawa 253-0042, Japan.
4) Present address: Research Institute for Production Development; 15 Shimogamo Morimoto-cho, Sakyo-ku, Kyoto 606-0805, Japan.

5) Fujita T., Inoue K., Yamamoto S., Ikumoto T., Sasaki S., Toyama R., Chiba K., Hoshino R., Okumoto T., J. Antibiot., 47, 208-215 (1994).

6) Kluepfel D., Bagli J., Baker H., Charest M.-P., Kudelski A., Sehgal S N., Vezina C., J. Antibiot., 25, 109-115 (1972).

7) Bagli J. F., Kluepfel D., St-Jacques M., J. Org. Chem., 38, 1253-1260 (1973).

8) Craveri R., Manachini P. L., Aragozzini F., Experientia, 28, 867-868 (1972).

9) Aragozzini F., Manachini P. L., Craveri R., Rindone B., Scolastico C., Tetrahedron, 28, 5493-5498 (1972).

10) Fujita T., Hirose R., Yoneta M., Sasaki S., Inoue K., Kiuchi M., Hirase S., Chiba K., Sakamoto H., Arita M., J. Med. Chem., 39, 4451- 4459 (1996).

11) Adachi K., Kohara T., Nakao N., Arita M., Chiba K., Mishina T., Sasaki S., Fujita T., Bioorg. Med. Chem. Lett., 5, 853-856 (1995).

12) Kiuchi M., Adachi K., Kohara T., Minoguchi M., Hanano T., Aoki Y., Mishina T., Arita M., Nakao N., Ohtsuki M., Hoshino Y., Teshima K., Chiba K., Sasaki S., Fujita T., J. Med. Chem., 43, 2946-2961 (2000).

13) Fujita T., Farumashia, 33, 591-593 (1997).

14) Kiuchi M., Farumashia, 43, 673-677 (2007).

15) Mandala S., Hajdu R., Bergstrom J., Quackenbush E., Xie J., Milligan J., Thornton R., Shei G.-J., Card D., Keohane C., Rosenbach M., Hale J., Lynch C. L., Rupprecht K., Parsons W., Rosen H., Science, 296, 346-349 (2002)

16) Brinkmann V., Davis M. D., Heise C. E., Albert R., Cottens S., Hof R., Bruns C., Prieschl E., Baumruker T., Hiestand P., Foster C. A., Zollinger M., Lynch K. R., J. Biol. Chem., 277, 21453-21457 (2002).

17) Chiba K., Yanagawa Y., Masubuchi Y., Kataoka H., Kawaguchi T., Ohtsuki M., Hoshino Y., J. Immunol., 160, 5037-5044 (1998).

18) Yanagawa Y., Sugahara K., Kataoka H., Kawaguchi T., Masubuchi Y., Chiba K., J. Immunol., 160, 5493-5499 (1998).

19) Matsuura M., Imayoshi T., Okumoto T., Int. J. Immunopharmacol., 22 , $323-331(2000)$

20) Kohno T., Tsuji T., Hirayama K., Watabe K., Matsumoto A., Kohno T., Fujita T., Biol. Pharm. Bull., 27, 1392-1396 (2004).

21) Kohno T., Tsuji T., Hirayama K., Iwatsuki R., Hirose M., Watabe K., Yoshikawa H., Kohno T., Matsumoto A., Fujita T., Hayashi M., Biol. Pharm. Bull., 28, 736-739 (2005).

22) Budde K., Schmouder R. L., Brunkhorst R., Nashan B., Lucker P. W. Mayer T., Choudhury S., Skerjanec A., Kraus G., Neumayer H. H., J. Am. Soc. Nephrol., 13, 1073-1083 (2002).

23) Kahan B. D., Karlix J. L., Ferguson R. M., Leichtman A. B., Mulgaonkar S., Gonwa T. A., Skerjanec A., Schmouder R. L., Chodoff L., Transplantation, 76, 1079-1084 (2003).

24) Tedesco-Silva H., Mourad G., Kahan B. D., Boira J. G., Weimar W., Mulgaonkar S., Nashan B., Madsen S., Charpentier B., Pellet P., Vanrenterghem Y. Transplantation, 77, 1826-1833 (2004).

25) Salvadori M., Budde K., Charpentier B., Klempnauer J., Nashan B., Pallardo L. M., Eris J., Schena F. P., Eisenberger U., Rostaing L., Hmissi A., Aradhye S., Am. J. Transplant., 12, 2912-2921 (2006).

26) Tedesco-Silva H., Pescovitz M. D., Cibrik D., Rees M. A., Mulgaonkar S., Kahan B. D., Gugliuzza K. K., Rajagopalan P. R., Esmeraldo R. de M., Lord H., Salvadori M., Slade J. M., Transplantation, 82, 16891697 (2006).

27) Kappos L., Antel J., Comi G., Montalban X., O’Connor P., Polman C. H., Haas T., Korn A. A., Karlsson G., Radue E. W., N. Engl. J. Med., $3551124-1140$ (2006).

28) Fujita T., Sasaki S., Yoneta M., Mishina T., Adachi K., Chiba K., PCT/WO94/08943.

29) Hirase S., Sasaki S., Hirose R., Yoneta M., Fujita T., PCT/WO99/1419.

30) Durand P., Peralba P., Sierra F., Renaut P., Synthesis, 2000, 505-506 (2000).

31) Kalita B., Barua N. C., Bezbarua M., Bez G., Synlett, 2001, 14111414 (2001).

32) Seidel G., Laurich D., Fürstner A., J. Org. Chem., 69, 3950-3952 (2004).

33) Sugiyama S., Arai S., Kiriyama M., Ishii K., Chem. Pharm. Bull., 53, $100-102(2005)$.

34) Pogosyan G. M., Zhamkochyan G. A., Matsoyan S. G., Armyanskii Khimicheskii Zhurnal, 22, 908-914 (1969). 